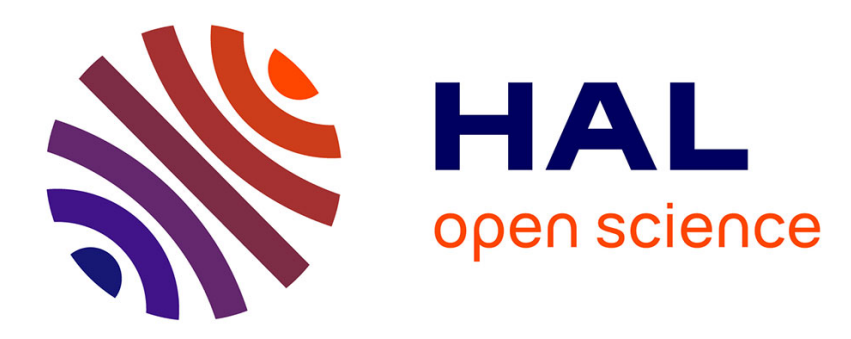

\title{
Critical Behavior of a Mixed Ising Ferrimagnet
}

G. Buendía, M. Novotny

\section{To cite this version:}

G. Buendía, M. Novotny. Critical Behavior of a Mixed Ising Ferrimagnet. Journal de Physique IV Proceedings, 1997, 07 (C1), pp.C1-175-C1-176. 10.1051/jp4:1997163 . jpa-00255110

\section{HAL Id: jpa-00255110 https://hal.science/jpa-00255110}

Submitted on 1 Jan 1997

HAL is a multi-disciplinary open access archive for the deposit and dissemination of scientific research documents, whether they are published or not. The documents may come from teaching and research institutions in France or abroad, or from public or private research centers.
L'archive ouverte pluridisciplinaire HAL, est destinée au dépôt et à la diffusion de documents scientifiques de niveau recherche, publiés ou non, émanant des établissements d'enseignement et de recherche français ou étrangers, des laboratoires publics ou privés. 


\title{
Critical Behavior of a Mixed Ising Ferrimagnet
}

\author{
G.M. Buendía and M.A. Novotny* \\ Departamento de Fisica, Universidad Simón Bolívar, Apdo. 89000, Caracas 1080, Venezuela \\ * Supercomputer Computations Research Institute, Florida State University and Department of Electrical \\ Engineering, Florida A\&M, Florida State University, Tallahassee, Florida 32310-6046, U.S.A.
}

\begin{abstract}
The critical behavior of a 2-D mixed ferrimagnetic spin2-spin1/2 Ising model has been studied with Monte Carlo and transfer-matrix techniques. This model is relevant for understanding bimetallic molecular ferrimagnetic materials. We calculate the finite temperature phase diagram putting special attention to the location and characterization of compensation points. We found that a compensation temperature appears when the interaction between the next-nearest neighbors spins $1 / 2$ is included.
\end{abstract}

\section{INTRODUCTION}

Ferrimagnetic ordering seems to play a crucial role in the behavior of a numerous group of molecular materials that are currently being synthesized by several experimental groups [1]. The different temperature dependence of the sublattices magnetizations in a ferrimagnet raises the interesting possibility of the existence of compensation points: temperatures where the resultant magnetization vanishes before the critical temperature is reached. The behavior of the compensation point has important technological applications particularly in the field of thermomagnetic recording.

Mixed Ising systems provide simple but interesting models to study ferrimagnetism. Mean and effective field theories predict the existence of tricritical and compensation points in mixed Ising systems that include only nearest-neighbors and crystal field interactions [2]. However, recent results based in non-perturbative techniques such as Monte Carlo and numerical transfermatrix indicate that these predictions are not reliable, and strongly suggest that the existence of compensation points is associated with second order interactions [3][4]. In this article we applied Monte Carlo and transfer-matrix numerical techniques to study a mixed Ising system in which spins $S( \pm 2, \pm 1,0)$ are intercalated in a square lattice with spins $\sigma( \pm 1 / 2)-$ spin2spin 1/2 model -. In a previous work it was shown that if only nearest-neighbors interactions and the crystal field are included, this model has no compensation point and no evidence of a tricritical point (predicted by mean-field theories) [4]. In this article we are going to study the effect of second-order interactions in the finite temperature phase diagram particularly in the location of the compensation point.

\section{THE MODEL}

The Hamiltonian of our model is the following

$$
H=-J_{1} \sum_{<n n>} S_{i} \sigma_{j}-J_{2} \sum_{\varangle n>} S_{i}^{2} \sigma_{j}-J_{3} \sum_{\varangle n n>>} S_{i} S_{l}-J_{4} \sum_{<n n n>} \sigma_{j} \sigma_{k}-h \sum_{i, j}\left(S_{i}+\sigma_{j}\right)+D \sum_{i} S_{i}^{2}
$$

where, $\mathrm{J}_{1}, \mathrm{~J}_{2}, \mathrm{~J}_{3}$ and $\mathrm{J}_{4}$ are the exchange interaction parameters, $\mathrm{h}$ is the external field, and $\mathrm{D}$ is the crystal field, all in energy units. In order to have an antiferromagnetic coupling between nearest neighbors $J_{1}$ is chosen to be negative. The exact ground state diagrams and the critical temperatures for the $\mathrm{J}_{1}-\mathrm{D}$ model (for which the only parameters in the Hamiltonian different from zero are $J_{1}$ and $D$ ) have been published elsewhere [4] . Mean-field theory predicts that the $J_{1}-D$ model where $S= \pm 1,0$ and $\sigma= \pm 1 / 2$, - spin1-spin1/2 model - has a compensation point, but Monte Carlo and transfer matrix results for both, the spin2spin1/2 and spin1-spin1/2 cases, show that there is no compensation point for the $J_{1}-\mathrm{D}$ model. For the spin1-spin $1 / 2$ model it was shown that the compensation point appears only when the interaction $\mathbf{J}_{4}$ is included [3]. We are interested to see if this is also the case for the spin2-spin $1 / 2$ model presented here. 


\section{NUMERICAL TECHNIQUES}

\subsection{The Monte Carlo (MC) method}

Data were obtained for a $60 \times 60$ square lattice with $10^{5} \mathrm{MC}$ steps per site after discarding the first $10^{3}$ steps per site. Configurations were generated by sequentially sweeping through the lattice and flipping the spins one at a time according with the heat-bath algorithm. The critical temperatures, $T_{c r i t}$, were calculated by locating the maximum value of the specific heat. The compensation points, $T_{\text {comp }}$, were located by finding the crossing point between the absolute values of the sublattice magnetizations such that,

$\left.\left|M_{1}\left(T_{\text {comp }}\right)\right|=\mid M_{2}\left(T_{\text {comp }}\right)\right), \quad \operatorname{sign}\left(M_{1}\left(T_{\text {comp }}\right)=-\operatorname{sign}\left(M_{2}\left(T_{\text {comp }}\right)\right)\right.$ and $T_{\text {comp }}<T_{\text {crit }}$

\subsection{Transfer-Matrix (TM) calculations}

Traditional numerical TM calculations were performed. The lattice was wrapped on a torus of finite width and infinite extent, and periodic boundary conditions were imposed. By calculating the ratio of the largest and next-largest eigenvalues of the TM and using the scaling form for the correlation length we obtain the finite strip estimates for the $T_{\text {crit }}$. The $T_{\text {comp }}$ was obtained by looking at the temperature below $\mathrm{T}_{\text {crit }}$ at which the magnetization operator (calculated using the two largest eigenvectors of the TM) is zero.

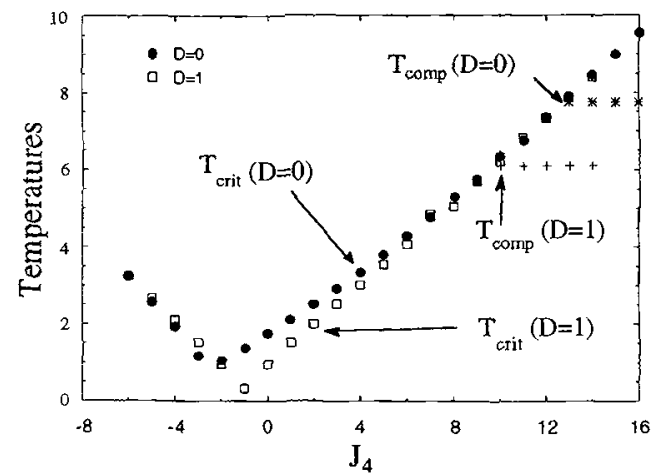

Fig.1: Critical and compensation temperature .vs. $\mathbf{J}_{4}$ for $D=0$ and $D=1$.

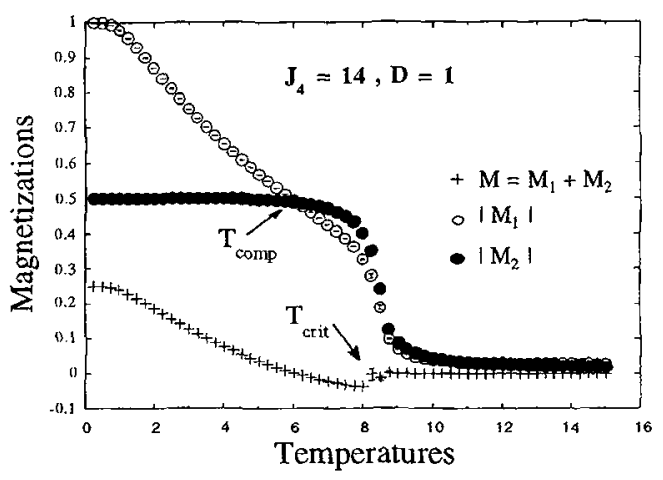

Fig.2: Total magnetization (M) and absolute values of the sublattice magnetizations, $\left|\mathrm{M}_{1}\right|,\left|\mathrm{M}_{2}\right|$.vs. temperature

\section{RESULTS AND CONCLUSIONS}

We find an excellent agreement between MC and TM results. The results indicate that a compensation point can not be induced in this model by nearest-neighbor interactions $\left(J_{1}-J_{2}-h-D\right)$, even when the second order interaction $J_{3}$ is included, we still do not find a compensation point. The compensation point appears when the $J_{4}$ interaction, between the $\sigma$ spins, is added ( $J_{1}{ }^{-} J_{4}-$ $D$ model). In Fig 1. we show $T_{\text {crit }}$ and $T_{\text {comp }}$ as funtions of $J_{4}$ for a couple of values of $D$. Notice that the compensation temperature does not exist until the $\mathrm{J}_{4}$ interaction takes a minimum positive value. This minimum value of $\mathrm{J}_{4}$ decreases with increasing values of $D$. The $T_{\text {crit }}$ always increases with $J_{4}>0$, but once $T_{\text {comp }}$ appears it remains independent of $J_{4}$, this behavior is expected, increasing $\mathrm{J}_{4}$ above the minimum have the effect of keeping the $\sigma$ sublattice ordered at higher temperatures (increasing $T_{\text {crit }}$ ) but has no effect on the $T_{\text {comp }}$ that has already been reached when the $S$ sublattice (weakly dependent on $\mathrm{J}_{4}$ ) magnetization was equal but of opposite sign to the $\sigma$ sublattice magnetization. Fig 2 . shows an example were the distinctive behavior of the magnetization at $T_{\text {crit }}$ and $T_{\text {comp }}$ can be observed. At $T_{\text {comp }}$ the sublattice magnetizations cancel each other, whereas at $T_{c r i t}$ both go to zero independently. The dependence of the compensation point with the longer range interactions has already be found experimentally [5].

\section{References}

[1] T. Mallah, S. Tiebaut, M. Verdaguer, and P. Veillet, Science 262 (1993) 1554.

[2] T. Kaneyoshi and M. Jascur, J. Magn. Magn. Mat 118 (1993) 17; T. Kaneyoshi, Physica A 205 (1994) 677.

[3] G. M. Buendía, M. A. Novotny and J. Zhang "Computer study of a d=2 mixed Ising ferrimagnet", Computer Simulations in Condensed Matter Physics VII, Athens 1994, D. Landau, K. Mon and H. Schuttler, Eds. (Springer, Berlin 1994) p223; G. M. Buendía and M. A. Novotny, FSU-SCRI preprint $95-15$, submitted to publication.

[4] G. M. Buendía and J. A. Liendo. USB preprint, submitted to publication.

[5] L. Ertl, G. Endl, and H. Hoffmann, J. Magn. Magn. Mat. 113 (1992) 227. 